\title{
$A$ randomized trial of nicotinamide and vitamin $E$ in children with recent onset type 1 diabetes (IMDIAB IX)
}

\author{
A Crinò, R Schiaffini ${ }^{1}, \mathrm{~S}$ Manfrini ${ }^{1}, \mathrm{C}$ Mesturino ${ }^{1}, \mathrm{~N}$ Visalli ${ }^{1}, \mathrm{G}$ Beretta Anguissola ${ }^{1}, \mathrm{C} \mathrm{Suraci}^{2}, \mathrm{D} \mathrm{Pitocco}^{3}$,
} $S_{S}$ Spera $^{1}$, S Corbi ${ }^{1}$, M C Matteoli, I P Patera, M L Manca Bitti ${ }^{4}$, C Bizzarri and P Pozzillion behalf of the IMDIAB group

Ospedale Bambino Gesù, Piazza S. Onofrio 4, 00163 Roma, ${ }^{1}$ Università Campus, Bio-Medico, via longoni 83, O0155 Roma, ${ }^{2}$ Ospedale S. Eugenio, via Rhodesia 18, 800154 Roma, ${ }^{3}$ Università Cattolica, Largo Agostino Gemelli 8, 00163 Roma, ${ }^{4}$ Università Tor Vergata, Via di Tor Vergata, 00133 Roma, Italy

(Correspondence should be addressed to P Pozzilli, Department of Endocrinology and Diabetes, University Campus Bio-Medico, Via E Longoni, 83, O0155 Rome, Italy; Email: p.pozzilli@unicampus.it)

\begin{abstract}
Objective: Various adjuvant therapies have been introduced along with intensive insulin therapy in patients with recent onset type 1 diabetes. Nicotinamide (NA), administered at diagnosis of the disease, can have beneficial effects on the clinical remission rate, improve metabolic control and preserve or slightly increase beta-cell function, probably by reducing toxicity due to free oxygen radicals. Vitamin E, a known antioxidant, inhibits lipid peroxidation; this can lead to protection of islet beta cells from the combined effects of interleukin 1, tumor necrosis factor and gamma interferon. The aim of the present study was to investigate whether the addition of vitamin E to NA could improve metabolic control and the residual beta-cell function, as measured by C-peptide secretion, in children and adolescents with recent onset type 1 diabetes; patients were followed-up for 2 years after diagnosis. Patients and study design: Recent onset type 1 diabetes patients $(n=64$, mean age 8.8 years) were recruited by participating centres of the IMDIAB group. Thirty-two patients were randomized to NA $(25 \mathrm{mg} / \mathrm{kg}$ body weight) plus vitamin $\mathrm{E}(15 \mathrm{mg} / \mathrm{kg}$ body weight); 32 patients acted as controls and received NA only at the same dose as above. Intensive insulin therapy was applied to both treatment groups.

Results: There were three drop outs during the 2-year follow-up period. Overall, patients assigned to the NA + vitamin E group or the NA group did not significantly differ in terms of glycated hemoglobin (HbA1c) levels, insulin requirement or baseline C-peptide secretion. Patients diagnosed at an age of less than 9 years showed significantly reduced C-peptide levels compared with those aged over 9 years at diagnosis and at the 2-year follow-up but there were no differences between the NA and $\mathrm{NA}+$ vitamin E treated groups. However at 6 months, patients over 9 years of age treated with NA + vitamin $\mathrm{E}$ showed significantly higher $C$-peptide compared with the NA group $(P<0.003)$. In both age groups and in the different treatment groups, C-peptide levels found at diagnosis were preserved 2 years later.

Conclusions: The use of NA alone, or in combination with vitamin E, along with intensive insulin therapy is able to preserve baseline C-peptide secretion for up to 2 years after diagnosis. This finding is of particular interest for pre-pubertal children with type 1 diabetes and has never been reported before.
\end{abstract}

European Journal of Endocrinology 150 719-724

\section{Introduction}

Type 1 diabetes is most probably caused by an autoimmune destruction of insulin-producing beta cells mediated by cytotoxic $\mathrm{T}$ cells recognizing specific cell associated antigens. The introduction of intensive insulin therapy at clinical diagnosis of the disease has been shown to preserve beta-cell mass up to 1 year after disease diagnosis, with deterioration of function soon after $(1-3)$. Various adjuvant therapies have been introduced along with intensive insulin therapy with the intention of protecting residual beta cells, although the results have been partially disappointing (4-6).

Nicotinamide (NA) has been shown, when administered at diagnosis of the disease, to have beneficial effects on the clinical remission rate, to improve metabolic control and to preserve or slightly increase betacell function (7). Possible mechanisms of action include: reducing cytokine cytotoxicity (8); improving insulin synthesis by increasing intracellular NAD content (9), thereby promoting beta-cell regeneration; preventing the toxic effect of nitric oxide on islets (10) and 
abolishing the inhibitory effect of interleukin $1 \beta$ on insulin release (11).

Vitamin E, a known antioxidant, inhibits lipid peroxidation and interferes with the process of cytokine-mediated cytotoxicity by acting as a free oxygen scavenger $(12,13)$. It has also been shown that lipid peroxidation is involved in the cytotoxic effects of cytokines on rat islets and that the inhibition of this process protects islet beta cells from the combined cytotoxic effects of interleukin 1, tumor necrosis factor and interferon gamma (14). Furthermore, vitamin E has been shown to be effective in preventing severe complications in long-standing type 1 diabetic patients (15). Vitamin E was used in one previous trial at diagnosis of type 1 diabetes with modest results (16).

Since the mechanisms of action of NA and vitamin E are different, the combination of these two vitamins may elicit interesting effects as they tackle the problem of islet-beta-cell cytotoxicity in diverse ways.

Considering the above reports and our previous encouraging experience using NA alone, the aim of the present study was to investigate, in patients with recent onset type 1 diabetes, the effect of the combination of NA + vitamin E or NA alone (when administered from diagnosis and continued for up to 2 years) on metabolic control and residual beta-cell function as measured by C-peptide secretion.

\section{Patients and methods}

\section{Study design and treatment protocol}

The primary aim of this trial was to find out whether the combination therapy of NA + vitamin E implemented at diagnosis in association with intensive insulin therapy improves metabolic control (insulin requirement, glycated hemoglobin (HbA1c)) and beta-cell function (C-peptide) compared with patients receiving NA alone.

\section{Sample size and statistical analysis}

The number of patients to be included in the study was calculated from the results of our previous trials and other trials in a population of patients of similar ages to those recruited for the present trial (courtesy of the International Diabetes Immunotherapy Group (IDIG) registry). The trial end-point was set as an increase of baseline C-peptide of $0.13 \mathrm{nM}$ at 6 months after diagnosis. Setting alpha (probability of type I error) equal to 0.05 and beta (probability of type II error) equal to $90 \%$, the required sample size was 54 patients for a two-sided test. To ensure the appropriate sample size, 64 patients were recruited to allow for drop outs.

After baseline measurements were completed, a permuted-block design was used to assign patients to each of the two treatment groups. A random number table was adopted with a prepared list, and a randomization code was assigned to each participating center.
The study was approved by the Ethical Committee of Università Campus Bio-Medico and informed consent was signed by parents.

\section{Selection of patients}

Patients with recent onset type 1 diabetes were recruited by participating centers of the IMDIAB group. Patients' inclusion criteria were the following: (a) diagnosis of the disease according to recent American Diabetes Association (ADA) guidelines; (b) age at presentation between 4 and 18 years; (c) duration of clinical disease (since the beginning of insulin therapy) $<4$ weeks; (d) no medical contra-indications or any other major chronic disease; (e) willingness and capability to participate in regular follow-up. In all patients, presence of autoantibodies to glutamic acid decarboxylase (GAD) confirmed diagnosis of type 1 diabetes.

The mean age of patients was 8.8 years. Since Tanner stage data were not available, a cut-off age of 9 years was considered appropriate for subsequent analysis of patients regarding puberty, with those under 9 years old being defined as pre-pubertal. Accordingly there were 38 patients under 9 years of age at diagnosis (mean age ( \pm S.D.) $6.3 \pm 1.2$ years) and 23 patients over 9 years old (mean age ( \pm S.D.) $12.8 \pm 7.1$ years). Of the former, 20 were treated with NA and 18 with NA + vitamin E. Of those patients over 9 years of age, 12 received NA alone and 11 received the combination of NA + vitamin E.

\section{Treatment protocol}

Thirty-two patients (mean age (土S.D.) $9 \pm 5.4$ years) were treated with NA ( $25 \mathrm{mg} / \mathrm{kg}$ body weight) and vitamin E (15 mg/kg body weight) and 32 patients (mean age ( \pm S.D.) $8.7 \pm 5.3$ years) were treated with the same dose of NA only. This dose was chosen based upon our previous experience (4). In all patients, adjuvant therapy with NA+vitamin E or NA began within 4 weeks of diagnosis. None of the eligible patients refused randomization. Patients were treated with intensive insulin therapy in order to optimize metabolic control as soon as possible after diagnosis and to maintain blood glucose levels to as near normal as possible.

\section{Guidelines for insulin therapy}

All patients were placed on three injections of regular insulin at meal times and one of neutral protamine hagedorn (NPH) insulin at bed time. Participating centers used the same treatment protocol as in previous IMDIAB trials (4) based on the following rules: if preprandial blood glucose values were below $6.5 \mathrm{mmol} / \mathrm{l}$, the insulin dose was decreased by $10 \%$; if blood glucose concentrations were consistently below $4.5 \mathrm{mmol} / \mathrm{l}$ for more than 3 days the insulin dose was decreased by $20 \%$. Insulin therapy was not discontinued unless 2-h 
post-prandial blood glucose concentrations measured at home were consistently below $7.5 \mathrm{mmol} / \mathrm{l}$. Patients with blood glucose concentrations above $10 \mathrm{mmol} / \mathrm{l}$ received a $10 \%$ increase in insulin dose or had their insulin regimen modified. Frequent (weekly) telephone consultations were arranged with patients to adjust the insulin dose as required.

\section{Investigations and follow-up}

Patients included in the study were followed up by the staff of the center where they were enrolled. Patients were started on a 55\% carbohydrate diet and received three to four injections daily of regular plus intermediate insulin. Each patient recorded daily capillary glucose concentration at fasting and before and after meals, for a total of at least 30 measurements weekly. The subcutaneous insulin dose was adjusted to obtain near-normal blood glucose concentrations.

Patients were examined weekly for the first month of therapy and then tri-monthly by the same team of physicians in each participating center for up to 2 years. Drug toxicity was evaluated at follow-up visits by liver and renal function tests and total blood counts. Glycated hemoglobin (normal range 4-7\%) was measured every 3 months by a column assay (Bio-Rad, Milan, Italy), and basal C-peptide concentration was evaluated after euglycemia was achieved before entry into the trial and at tri-monthly intervals for 2 years thereafter. C-peptide was measured by radioimmunoassay using a commercially available kit (Bio-Rad). The normal range of fasting C-peptide established in 150 control subjects (71 females and 79 males, aged 5-40 years, median 18 years, with no family history of type 1 diabetes) was 0.35-1 mmol/l with intracoefficients and intercoefficients varying between 10 and $15 \%$ respectively.

\section{Statistical analysis}

Data were analyzed using the Statsoft statistical package for IBM computers. Analysis of variance, $\chi^{2}$ and $t$-test were applied where appropriate. In order to analyze possible effects of puberty on metabolic parameters, comparisons were carried out between patients treated with NA and NA + vitamin E irrespective of age at diagnosis, as well as dividing patients into those under 9 years old at diagnosis and those over 9 years old at diagnosis.

\section{Results}

There were three drop outs (one in the NA + vitamin E group and two in the NA group), as a result of changes in residential location. The results of this study are shown in Figs $1-3$. During the 2-year evaluation period, patients assigned to NA + vitamin E or NA treatment did not significantly differ in terms of HbA1c levels $(P=$ n.s. $)$. Mean HbAlc levels were between 6 and $7 \%( \pm 1$ S.D. $)$ at each time point measured, indicating that good metabolic control was achieved and maintained (Fig. 1). To reach such control, patients treated with NA+vitamin E received slightly less insulin than patients on NA alone; this was significant only at 18 months $(P<0.03)$ (Fig. 2). For the NA group, the insulin requirement at 3 months was $0.48 \pm 0.27 \mathrm{IU} / \mathrm{kg}$ per day which gradually increased to reach $0.90 \pm 0.24 \mathrm{IU} / \mathrm{kg} /$ day after 24 months. For the NA + vitamin E group, at 3 months the insulin requirement was $0.40 \pm 0.26 \mathrm{IU} / \mathrm{kg}$ per day gradually increasing to $0.75 \pm 0.29 \mathrm{IU} / \mathrm{kg}$ per day after 24 months. Although the differences were not statistically significant, there appears to be a general trend that after 18 months, the NA group may require slightly more insulin than the NA + vitamin E group.

The overall data on baseline C-peptide secretion are shown in Fig. 3. Over the 2-year follow-up there were no significant differences in C-peptide levels between the two groups. Data were then analyzed according to the age at diagnosis as this parameter may influence residual beta-cell function. Overall, patients aged less

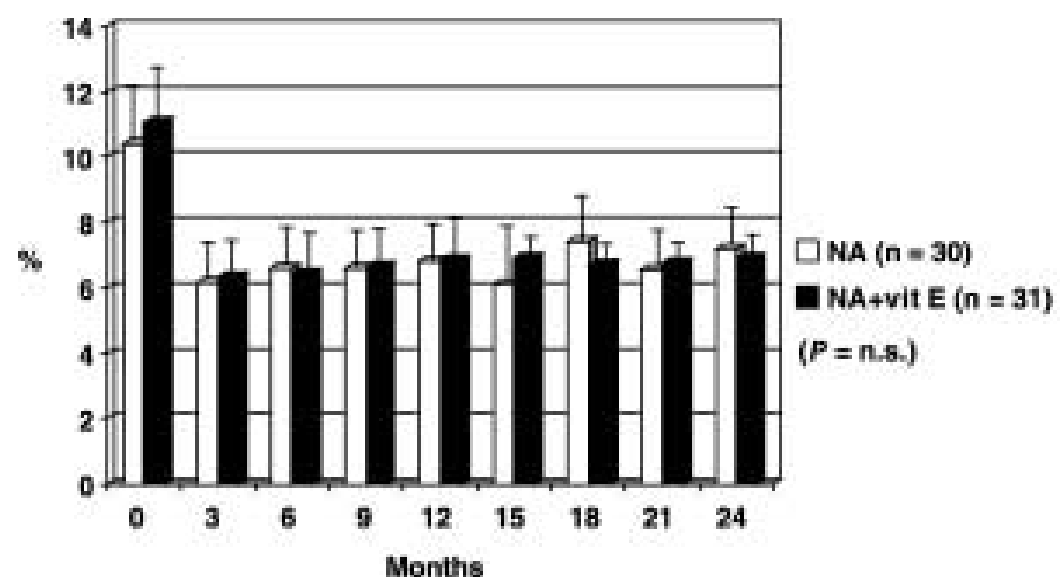

Figure 1 Mean percentage HbAlc (士s.D.) in the two treatment groups over a 2-year follow-up period. No significant differences were observed between treatment groups at any time point. 


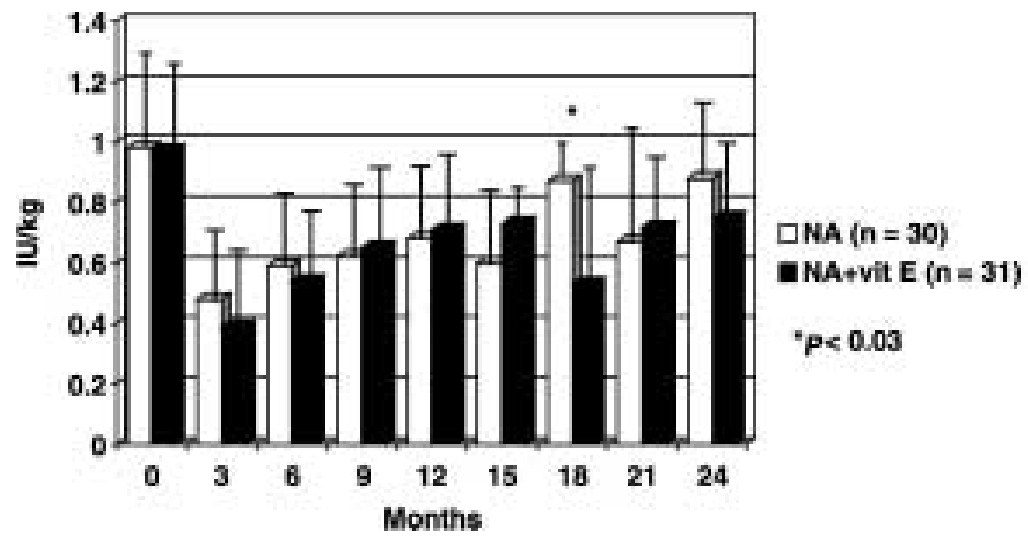

Figure 2 Mean insulin requirement ( \pm S.D.) in the two treatment groups over the 2-year follow-up period. $P<0.03$ (Mann-Whitney $U$ test) at 18 months. There were no differences between the treatment groups at other time points.

than 9 years at diagnosis showed significantly reduced C-peptide levels at entry to the trial compared with patients aged over 9 years $(0.22 \pm 0.2$ vs $0.32 \pm 0.2 \mathrm{nM}$ respectively (means \pm S.D.),$\quad P<0.01$ ); similar results were seen at 24 months $(0.23 \pm 0.1$ vs $0.36 \pm 0.2 \mathrm{nM}$ respectively (means \pm S.D.), $P<0.01$ ).

Regarding the type of treatment in patients diagnosed at under 9 years of age, no significant differences were observed over the study period except at 6 months when the NA + vitamin E group showed significantly higher $\mathrm{C}$-peptide levels compared with the NA group $(0.40 \pm 0.2$ vs $0.21 \pm 0.1 \mathrm{nM}$ respectively, $P<0.003)$. In patients diagnosed at over 9 years of age, NA alone was able to increase $C$-peptide levels significantly compared with the NA + vitamin E group at 6 months $(0.43 \pm 0.2$ vs $0.27 \pm 0.1 \mathrm{nM}$ respectively, $P<0.01)$ and at 9 months $(0.43 \pm 0.2$ vs $0.25 \pm 0.2 \mathrm{nM}$ respectively, $P<0.04)$. No differences in C-peptide levels at 2 years were observed between the different treatment groups.

When $\mathrm{HbA} 1 \mathrm{c}$ values and insulin requirement were evaluated according to age at diagnosis, no significant differences were observed between age groups (data not shown).

Overall the main finding is that, no decline in C-peptide levels was observed at 2 years (compared with levels at diagnosis), irrespective of age. Finally, no adverse effects were reported in patients of both groups over the follow-up period. Most importantly, episodes of severe hypoglycemia were limited and were not significantly different between the two treated groups.

\section{Discussion}

In this randomized trial we found that the use of NA alone or in combination with vitamin E, together with intensive insulin therapy, was able to preserve baseline C-peptide secretion for up to 2 years after diagnosis. This parameter has important implications for disease outcome (17-19). Our trial is the first one that has prolonged the adjuvant therapy using these compounds for 2 years to evaluate the natural history of beta-cell function in a group of children and adolescents with recent onset type 1 diabetes. The observation that baseline C-peptide secretion can be maintained for 2 years after diagnosis in patients diagnosed before and after puberty is novel since all previous trials published studying this particular pre-pubertal age group of patients have shown a significant reduction of baseline and/or stimulated C-peptide at 1 year after diagnosis (20-23).

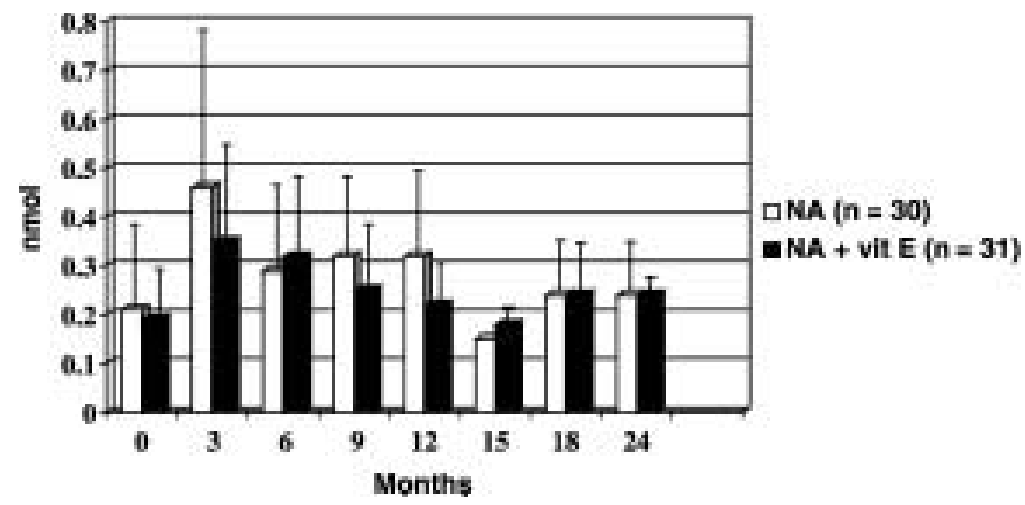

Figure 3 Mean C-peptide secretion ( \pm S.D.) in the two treatment groups over the 2-year follow-up period. No significant differences were observed between treatment groups at any time point. Note the mean C-peptide values at 2 years overlap those at diagnosis. 
In the present study we did not consider a randomized patient group where intensive insulin therapy only could have been implemented without the addition of NA or vitamin E since the main aim of this trial was to evaluate whether the combination of NA + vitamin E was more effective than NA alone in preserving beta-cell function. In another IMDIAB group study, patients with recent onset type 1 diabetes matched for age were followed up for 2 years with either intensive insulin therapy only or NA added to the treatment (24). In the latter group significantly more insulin was needed whereas $\mathrm{HbAlc}$ levels were higher in the group receiving intensive insulin therapy only; residual beta-cell function, assessed by C-peptide secretion, was similar between the two groups (21). This suggests that NA probably exerts its action not only by controlling the process of beta-cell destruction, but may also favor insulin resistance.

In the present IMDIAB IX trial the combination therapy of NA + vitamin E showed overall similar effects on metabolic control when compared with patients receiving NA alone. It should be noted that at some time points during the course of the study, patients receiving the combination therapy of NA + vitamin E required significantly less insulin than patients on NA alone to reach the same HbA1c levels. Interestingly at 6 months, patients diagnosed at less than 9 years of age showed a significant increase of C-peptide in the NA + vitamin E group compared with patients treated with NA only. In contrast, in older patients, NA alone appeared at 6 and 9 months after diagnosis to improve residual beta-cell function more than the combination therapy $\mathrm{NA}+$ vitamin E. Such changes were nevertheless minimal and at 2 years C-peptide levels were similar between the two treated groups. The notable observation is that baseline C-peptide at diagnosis clearly reflects the age of patients at the time of disease diagnosis, being lowest in pre-pubertal children.

Another relevant observation in this trial is that patients received intensive insulin treatment for the 2-year follow-up. Other investigators have employed a similar approach, however we believe that the administration of three injections of regular insulin at meal times and one NPH insulin at bed time do not, in isolation, constitute intensive insulin therapy. Adjustment of the insulin dose via continuous consultation with the patient and their parents is probably crucial. In this respect, frequent telephone consultations (weekly), repeated consultations (tri-monthly) continued for 2 years and the motivation of patients and physicians are all important features. Indirect evidence to support the relevance of intensive insulin therapy comes from the results of the IMDIAB VIII trial where recent onset type 1 diabetes patients were treated with continuous intensive insulin therapy from diagnosis (25). These patients showed a remarkable $38 \%$ increase in C-peptide secretion 2 years after diagnosis. It should be noted that more frequent and/or severe hypoglycemic episodes which may occur with such therapy were not observed in our studies.

An interesting aspect to consider is that the reduction in C-peptide secretion is usually taken as an end-point parameter for calculating the sample size in immune intervention trials. However, based on our findings, if appropriate intensive insulin therapy is adopted, the decline in C-peptide secretion (at least baseline) cannot be taken as a parameter for calculating the sample size in trials of this kind. Other parameters (probably immunological) should be identified for this purpose.

In conclusion, it is now well recognized that residual C-peptide secretion is associated with a reduced prevalence of late diabetic complications (3), therefore our aim with young patients with type 1 diabetes is to prolong, as far as possible, such secretion. It appears that this is now feasible, even in very young children, for at least 2 years after diagnosis using intensive insulin therapy. The use of NA alone or in combination with vitamin E, along with intensive insulin therapy, as shown in the present trial, is of particular interest especially for pre-pubertal children.

\section{Acknowledgements}

IMDIAB GROUP: Paolo Pozzilli, Natalia Visalli, Silvia Manfrini, Elvira Fioriti, Giusy Coppolino, Luciana Valente, Chiara Guglielmi, Giuseppina Beretta Anguissola, Flavia Costanza, Anna Lisa Montemari, Manon Khazrai Merola, Cesarea Leomanni, Maria C. Matteoli, I. Patrizia Patera, Marco Cappa, Antonio Crinò, Stefania Corbi, Sabrina Spera, Riccardo Schiaffini, Concetta Suraci, Marco Cervoni, Anna Cantagallo, Giancarlo De Mattia, Maria R. Cassone Faldetta, Maria L. Manca Bitti, Carla Bizzarri, Dario Pitocco and Giovanni Ghirlanda. This trial was supported by grants from CISD, MIUR $40 \%$ and the Italian Ministry of Health. The assistance of Dr Andrea Stoler in the preparation of the manuscript is greatly appreciated.

\section{References}

1 The Diabetes Control and Complications Trial Research Group. Effect of intensive therapy on residual $\beta$-cell function patients with type 1 diabetes in the diabetes control and complications trial. A randomised, controlled trial. Annual Review of Internal Medicine $1998 \mathbf{1 2 8} 517-523$.

2 Bolli GB. Physiological insulin replacement in type 1 diabetes mellitus. Experimental and Clinical Endocrinology/Diabetes 2001109 (Suppl 2) S317-S332.

3 The Writing Team for the Diabetes Control and Complications Trial/Epidemiology of Diabetes Interventions and Complications Research Group. Effect of intensive therapy on the microvascular complications of type 1 diabetes mellitus. Journal of the American Medical Association $2002 \mathbf{2 8 7} 2563-2569$.

4 Pozzilli P, Visalli N, Cavallo MG, Signore A, Baroni MG, Bozzetti R $\&$ the IMDIAB group. Vitamin $\mathrm{E}$ and nicotinamide have similar effects in maintaining residual beta-cell function in recent onset 
insulin-dependent diabetes (the IMDIAB IV study). European Journal of Endocrinology $1997137234-239$.

5 Visalli N, Cavallo MG, Signore A, Baroni MG \& Pozzilli P on behalf of the IMDAIB group. A multi-centre randomised trial of two different doses of nicotinamide in patients with recent-onset type 1 diabetes (The IMDIAB VI). Diabetes/Metabolism Research and Review $199915181-185$.

6 Pozzilli P, Pittocco D, Visalli N, Cavallo MG, Buzzetti R, Crinò A et al. and the IMDIAB group. No effect of oral insulin on residual beta-cell function in recent-onset type 1 diabetes (the IMDIAB VII). Diabetologia 200043 1000-1004.

7 Pozzilli P, Visalli N, Signore A, Baroni MG, Buzzetti R, Cavallo MG et al. (IMDIAB V) Double blind trial of nicotinamide in recent onset insulin dependent diabetes mellitus. Diabetologia 199538 $848-852$.

8 Yamada K, Miyajima F \& Nonaka K. Inhibition of cytokineinduced MHC class II, but not class I, molecule expression on mouse islet cells by nicotinamide and 3-aminobenzamide. Diabetes 199039 1125-1130.

9 Pociot F, Reimers JI \& Anderson HU. Nicotinamide - biological actions and therapeutic potential in diabetes prevention. Diabetologia $199336574-576$.

10 Kallman B, Burkart U, Krancke KD, Koib-Bachofen V \& Kolb H. Toxicity of chemical-generated nitric oxide towards pancreatic islet cells can be prevented by nicotinamide. Life Sciences 1992 $51671-678$.

11 Andersen RU, Jorgensen KR, Egenberg J, Mandrup-Poulsen T \& Nerup J. Nicotinamide prevents interleukin-1 effects on accumulated insulin release and nitric oxide production in rat islet of Langerhans. Diabetes 199443 770-777.

12 Paredes S, Girona J, Hurt-Camejo E, Vallve JC, Olive S, Heras M, Benito P \& Masana L. Antioxidant vitamins and lipid peroxidation in patients with rheumatoid arthritis: association with inflammatory markers. Journal of Rheumatology $2002292271-2277$.

13 Kagan VE, Kisin ER, Kawai K, Serinkan BF, Osipov AN, Serbinova EA, Wolinsky I \& Shvedova AA. Toward mechanism-based antioxidant interventions: lessons from natural antioxidants. Annual Review of the New York Academy of Sciences 2002959 188-198.

14 Rabinovitch A, Suarez WL, Thomas PD, Strynadka K \& Simpson I. Cytotoxic effects of cytokines on rat islets: evidence for involvement of free radicals and lipid peroxidation. Diabetologia 1992 35 409-413.

15 Rosen P \& Toeller M. Vitamin E in diabetes. Increased oxidative stress and its prevention as a strategy to prevent vascular complications? International Journal of Vitamin and Nutrition Reserach $199969206-212$.
16 Ludvigsson J, Samuelsson U, Johansson C \& Stenhammar L. Treatment with antioxidants at onset of type 1 diabetes in children: a randomized, double-blind placebo-controlled study. Diabetes/Metabolism Research and Review 200117 131-136.

17 Ludvigsson J \& Heding LG. C-peptide in children with juvenile diabetes. A preliminary report. Diabetologia $197612627-630$.

18 Ludvigsson J, Heding LG, Larsson Y \& Leander E. C-peptide in juvenile diabetics beyond the postinitial remission period. Relation to clinical manifestations at onset of diabetes, remission and diabetic control. Acta Paediatrica Scandinavica 197766 177-184.

19 Knip M \& Akerblom HK. Plasma C-peptide and insulin in neonates, infants, and children. Journal of Pediatrics 198199 103-105.

20 Lombardo F, Valenzise M, Wasniewska M, Messina MF, Ruggeri C, Arrigo T \& DeLuca F. Two-year prospective evaluation of the factors affecting honeymoon frequency and duration in children with insulin dependent diabetes mellitus: the key-role of age at diagnosis. Diabetes Nutrition and Metabolism 200215 246-251.

21 Buyukgebiz A, Cemeroglu AP, Bober E, Mohn A \& Chiarelli F. Factors influencing remission phase in children with type 1 diabetes mellitus. Journal of Pediatric Endocrinology and Metabolism 2001 14 1585-1596.

22 Bonfanti R, Bazzigaluppi E, Calori G, Riva MC, Viscardi M, Bognetti E et al. Parameters associated with residual insulin secretion during the first year of disease in children and adolescents with Type 1 diabetes mellitus. Diabetic Medicine 199815 844-850.

23 Peig M, Gomis R, Ercilla G, Casamitjana R, Bottazzo GF \& PujolBorrell R. Correlation between residual beta-cell function and islet cell antibodies in newly diagnosed type I diabetes. Followup study. Diabetes 198938 1396-1440.

24 Visalli N, Crinò A, Schiaffini R, Matteoli M, Patera P, Pozzilli P et al. on behalf of the IMDIAB group. C-peptide two years after diagnosis of Type 1 diabetes: the effect of nicotinamide and intensive insulin therapy. Diabetologia 200245 (Suppl 2) A119.

25 Pozzilli P, Crinò A, Schiaffini R, Manfrini S, Fioriti E, Coppolino G and the IMDIAB Group. A 2-year pilot trial of continuous subcutaneous insulin infusion versus intensive insulin therapy in patients with newly diagnosed type 1 diabetes (IMDIAB 8). Diabetes Technology E Therapy 20035 965-973.

Received 13 May 2003

Accepted 30 January 2004 